PTU-095 BIFIDOBACTERIUM SPECIES REDUCE LIPOPOLYSACCHARIDE-INDUCED SMALL INTESTINAL EPITHELIAL CELL SHEDDING IN VIVO IN A MYD88DEPENDENT MANNER AND PROTECT AGAINST DSS-INDUCED COLITIS

${ }^{1} \mathrm{~K}$ Hughes* ${ }^{1} \mathrm{C}$ Alcon-Giner, ${ }^{2} \mathrm{D}$ VanSinderen, ${ }^{1} \mathrm{~L}$ Hall, ${ }^{1} \mathrm{~A}$ Watson. ${ }^{1}$ Medicine, University of East Anglia, Norwich, UK; ${ }^{2}$ Alimentary Pharmabiotic Centre, University College Cork, Cork, Ireland

\subsection{6/gutjnl-2014-307263.169}

Introduction Cell shedding, the process by which intestinal epithelial cells (IECs) are extruded from the small intestinal (SI) villus is known to be elevated in patients with inflammatory bowel disease (IBD) and is correlated with disease relapse. Importantly, there is evidence that the gut bacterial communities (microbiota) influences intestinal epithelial function including gene expression, cell division and energy balance. We thus sought to determine whether specific members of the microbiota, 'probiotic' bifidobacterial species, modulate rates of cell shedding and progression of Dextran sodium sulphate (DSS)-mediated colitis.

Methods C57BL/6 mice (WT) or mice deficient in epithelial Myd88 (Vil-Cre +; Myd88 -/-) (Myd88 KO) were orally gavaged with $1 \times 10^{9}$ Bifidobacterium breve UCC2003, B. longum NCIMB8809 or PBS (control) in $3 \mathrm{x}$ daily doses. To induce SI cell shedding, mice were injected with $1.25 \mathrm{mg}$ kg-1 Lipopolysaccharide (LPS) intrapertioneally. Animals were euthanized $1.5 \mathrm{hr}$ post-LPS and SI tissue sections analysed for cleaved caspase 3 (CC3) by immunohistochemistry to score shedding along the first 50 cell positions from the villus tip. For colitis studies, control mice or mice colonised with $B$. breve were administered 2\% DSS in drinking water for 6 days and euthanized 8 days post-DSS. Disease activity index (DAI) was recorded daily and histology performed on formalin-fixed tissue sections including periodic acid/Schiff (PAS) stain (goblet cell stain).

Results Mice receiving B. breve and B. longum showed less CC3 + ve shedding cells $(3.6 \%+/-0.6, \mathrm{p}<0.001$ and $7.6 \%+/-2.9$, ns, respectively) compared to WT mice $(10.6 \%+/-1.3)$. Interestingly, the protective effect of $B$. breve was lost in $\mathrm{Myd} 88 \mathrm{KO}$ mice receiving LPS as numbers of CC3 +ve IECs were the same in mice receiving $B$. breve or vehicle control $(13.3 \%+/-1.7$ vs $10.4 \%+/ 1.3$; ns), indicating that the protective effect may be mediated by Toll-like receptors. In our colitis model, mice colonised with $B$. breve had reduced DAI compared to control mice, coupled with a significant increase in numbers of PAS + ve goblet cells per crypt $(8.2 \%+/ 1.6$ vs $16.0 \%+/-0.6 ; \mathrm{p}=0.05)$.

Conclusion Bifidobacterial species modulate a reduction in rates of cell shedding from the SI villus, potentially via the Myd88 signalling pathway. B. breve is also able to partially ameliorate the adverse effects of DSS-induced colitis through induction of goblet cells. In summary, bifidobacteria, particularly $B$. breve, may be beneficial as a therapeutic agent for IBD.

Disclosure of Interest None Declared.

\section{PTU-096 COST EFFICIENCY OF FAECAL CALPROTECTIN IN ASSESSING NEW REFERRALS WITH ALTERED BOWEL HABIT}

K Bundhoo*, A Aravinthan, K Lithgo, T Price, M Johnson. Gastroenterology, Luton and Dunstable Hospital, Luton, UK

10.1136/gutjnl-2014-307263.170
Introduction Altered bowel habits (ABH) is one of the commonest reasons for referral to the gastroenterology clinic. The spectrum of organic and functional bowel symptoms provides a diagnostic dilemma. Functional bowel disorders are common, occurring in $15-20 \%$ of Western populations. ${ }^{1}$ Therefore, it is important not to create an economic burden by overinvestigation.

Faecal calprotectin (FC) is a protein released from neutrophilic leucocytes into the intestinal lumen in response to mucosal inflammation. It is a well-validated, non-invasive test that can differentiate between organic and functional bowel disease with 93\% sensitivity and $96 \%$ specificity. ${ }^{2}$ These features make FC measurement a useful objective test in guiding further investigations.

Methods Over a 2 year period, all FC data was collected in new patients referred to the outpatient clinic for further assessment of $\mathrm{ABH}$ and where a diagnostic dilemma existed. Results were recorded as normal $(<50 \mu \mathrm{g} / \mathrm{g})$, borderline $(50-100 \mu \mathrm{g} / \mathrm{g})$ or positive $(>100 \mu \mathrm{g} / \mathrm{g})$ and correlated with the use of further endoscopic or radiological assessment. Department of Health $(\mathrm{DoH})$ tariffs were used to assess cost burden and potential savings.

Results 275 FC measurements were performed in new referrals where there was a dilemma about diagnosis or need for further investigation. Colonoscopy was spared in 71\% (196/275), including 139/164 normals, $16 / 22$ borderline and $35 / 89$ positives.

Despite a normal FC result, 25 patients underwent endoscopic investigation after initial assessment. Of these, 16 procedures were normal, 4 had diverticular disease and 2 had low grade dysplastic polyps. Some patients underwent CT colonography with positive findings in $4 / 17$ of the normal FC group (3 diverticular disease, 1 incidental gastric malignancy), 0/2 with borderline FC and 8/15 with positive FC measurement (5 diverticular disease, 1 suspected ileal ulcer, 2 cancers).

If all 275 patients had undergone colonoscopy the cost for the Clinical Commissioning Group (CCG) would be $£ 154275$. Risk stratifying with FC assessment reduced this to $£ 44319$, saving $£ 109956$.

Conclusion Faecal calprotectin assessment saved $71 \%$ of possible colonoscopies in those new patients assessed for $\mathrm{ABH}$ where there was a dilemma as to whether endoscopic investigation was necessary. This provided clinicians with the confidence to diagnose and manage functional bowel symptoms earlier. FC testing also saved our CCG $£ 109956$ of potentially unnecessary colonoscopy with the simultaneous advantage of reducing endoscopy waiting times.

\section{REFERENCES}

1 Drossman et al. Irritable bowel syndrome. A technical review for practice guideline development. Gastroenterology1997;112:2120-2137

2 Van Rheenen et al. Faecal calprotectin for screening of patients with suspected inflammatory bowel disease: diagnostic meta-analysis. BMJ 2010:341:c3369

Disclosure of Interest None Declared.

\section{PTU-097 THE BENEFITS OF USING FAECAL CALPROTECTIN AS A MONITORING TOOL TO ASSESS INFLAMMATORY BOWEL DISEASE AND PRE-EMPTIVELY UPREGULATE TREATMENT IN ASYMPTOMATIC PATIENTS}

K Bundhoo*, A Aravinthan, T Price, K Lithgo, M Johnson. Gastroenterology, Luton and Dunstable Hospital, Luton, UK

\subsection{6/gutjnl-2014-307263.171}

Introduction Calprotectin is an abundant neutrophil protein that is released during inflammation. The level of faecal calprotectin 